\title{
Treatment Patterns and Outcomes among Adults Admitted to Hospital in the United Kingdom (UK) due to Plaque or Erythrodermic Psoriasis \\ DOI:
}

10.1111/bjd.15270

\section{Document Version}

Accepted author manuscript

Link to publication record in Manchester Research Explorer

Citation for published version (APA):

Schaefer, C., Mamolo, C., Cappelleri, J. C., Daniel, S., Le, C., Tatulych, S., Griffiths, C. E. M., \& Hampton, P. J. (2017). Treatment Patterns and Outcomes among Adults Admitted to Hospital in the United Kingdom (UK) due to Plaque or Erythrodermic Psoriasis. The British journal of dermatology. https://doi.org/10.1111/bjd.15270

Published in:

The British journal of dermatology

\section{Citing this paper}

Please note that where the full-text provided on Manchester Research Explorer is the Author Accepted Manuscript or Proof version this may differ from the final Published version. If citing, it is advised that you check and use the publisher's definitive version.

\section{General rights}

Copyright and moral rights for the publications made accessible in the Research Explorer are retained by the authors and/or other copyright owners and it is a condition of accessing publications that users recognise and abide by the legal requirements associated with these rights.

\section{Takedown policy}

If you believe that this document breaches copyright please refer to the University of Manchester's Takedown Procedures [http://man.ac.uk/04Y6Bo] or contact uml.scholarlycommunications@manchester.ac.uk providing relevant details, so we can investigate your claim.

\section{OPEN ACCESS}


Received Date : 14-Mar-2016

Revised Date : 09-Nov-2016

Accepted Date : 21-Nov-2016

Article type : Research Letter

\section{Treatment Patterns and Outcomes among Adults Admitted to Hospital in the United Kingdom (UK) due to Plaque or Erythrodermic Psoriasis}

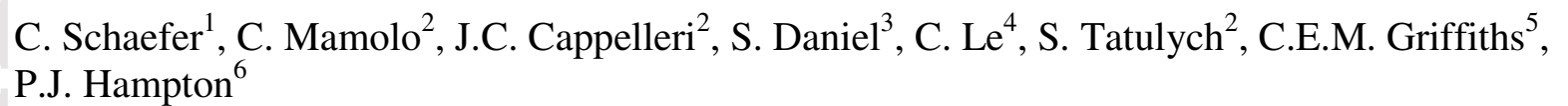

${ }^{1}$ Covance Market Access Services Inc, Gaithersburg, MD, USA

${ }^{2}$ Pfizer Inc, Groton, CT, USA

${ }^{3}$ Covance Market Access Services Inc, Conshohocken, PA, USA

${ }^{4}$ Covance Market Access Services Inc, San Diego, CA, USA

${ }^{5}$ Dermatology Centre, Salford Royal Hospital, University of Manchester, Manchester Academic Health Science Centre, Manchester, UK

${ }^{6}$ Newcastle Hospitals NHS Trust, Newcastle, UK

\section{Corresponding Author:}

Carla Mamolo, $\mathrm{PhD}$

Pfizer Inc.

Eastern Point Road

Groton, CT 06340 USA

Phone +1-860-441-3374

Email: carla.m.mamolo@pfizer.com

Funding Sources: This research was funded by Pfizer Inc.

Conflicts of Interest Disclosures: This research was supported by Pfizer Inc. C Schaefer, S Daniel, and C Le are employees of Covance Market Access Services Inc., which was engaged by Pfizer Inc. for study design, execution and analysis, and to develop this manuscript. C Mamolo, J Cappelleri, and S Tatulych are employees and shareholders of Pfizer Inc. CEM Griffiths is an employee of the University of Manchester and was an investigator for the study. He was not

This article has been accepted for publication and undergone full peer review but has not been through the copyediting, typesetting, pagination and proofreading process, which may lead to differences between this version and the Version of Record. Please cite this article as doi: $10.1111 /$ bjd. 15270

This article is protected by copyright. All rights reserved. 
financially compensated for collaborative efforts on publication-related activities. PJ Hampton is an employee of Newcastle Hospitals and was an investigator for the study. He was not financially compensated for collaborative efforts on publication-related activities.

Dear EDITOR: The National Institute for Health and Care Excellence (NICE) identified treatment of moderate-to-severe psoriasis as an area for research. ${ }^{1-2}$ No recent studies have assessed treatment patterns and outcomes among patients hospitalised for psoriasis in the $\mathrm{UK}^{3-5}$

This study evaluated clinician- and patient-reported outcomes, treatment patterns, and hospital length of stay (LOS) in patients admitted to hospital for plaque or erythrodermic psoriasis, and enrolled 61 eligible patients across 9 UK hospital sites from 15 November 2013 to 2 June 2014. Inclusion criteria included: $\geq 18$ years; willing and able to provide written informed consent; able to read, understand, and complete questionnaires; and admitted as an inpatient with plaque or erythrodermic psoriasis. Patients were ineligible if they had participated in an investigational drug trial in the previous 6 months; or had a serious/unstable medical or psychological condition that would compromise participation.

Study site personnel recorded eligibility criteria; patient clinical characteristics; clinicianreported outcomes, including Psoriasis Area Severity Index (PASI), Body Surface Area (BSA) affected by psoriasis, and Physician Global Assessment (PGA; 5-point scale) at admission and discharge; psoriasis treatments, and LOS. Patients reported psoriasis-related symptoms, health status (12-Item Short Form Health Survey, v2 [SF-12v2], EuroQoL 5-Dimensions, 3-Levels [EQ-5D-3L]), mood (Hospital Anxiety and Depression Scale [HADS]), productivity (Work Productivity and Activity Impairment [WPAI]), and dermatology-related quality-of-life

This article is protected by copyright. All rights reserved. 
(Dermatology Life Quality Index [DLQI]) at admission, and at discharge, employment status, psoriasis-related symptoms, EQ-5D-3L, and DLQI. Study materials were approved by the National Research Ethics Service Committee London-Stanmore (London, UK).

Descriptive statistics are reported among those responding to each item. Changes in continuous scores/outcomes from admission to discharge are assessed with a paired t-test and summarised for patients with both admission and discharge measures. Multivariate linear regression with the stepwise selection procedure was conducted to ascertain predictors of LOS with candidate predictors (age, gender, PASI score at admission, comorbidities present in $\geq 20 \%$ of the sample, duration of psoriasis, and previous hospitalisation). Statistical analyses were performed using $\mathrm{SAS}^{\circledR}$ version $9.3 .^{6}$

Mean age was 45.5 years; $50.8 \%$ were male. Mean time since psoriasis diagnosis was 20.0 years. Most $(78.7 \%)$ had $\geq 1$ previous psoriasis-related hospitalisations. Mean number of clinician-diagnosed comorbid conditions was 2.5, with psoriatic arthritis (34.4\%), depression (24.6\%), and arterial hypertension (21.3\%) the most commonly reported. At admission, mean scores indicated substantial impairment in health status, mood, and productivity (SF-12v2 physical and mental component summary: 35.4 and 32.1, respectively; HADS: 9.7 anxiety, 9.6 depression; WPAI: 68.7\% activity impairment, 79.2\% work impairment. (Table 1)

This article is protected by copyright. All rights reserved. 
Mean PASI improved from admission to discharge (25.2 to 12.1), as did EQ-5D-3L (0.34 to 0.60 ), total DLQI (20.1 to 12.0 ), and psoriasis symptom scores. (Table 2) For 35 patients with PASI scores at both admission and discharge, 18 (51.4\%) achieved a 50\% reduction in PASI (PASI50), and 8 (22.9\%) achieved a 75\% reduction in PASI (PASI75); $16(45.7 \%)$ had a PASI $>10$ at discharge. For 47 patients with a DLQI score at both time points, 28 (59.6\%) achieved $\mathrm{a} \geq 5$-point DLQI improvement from admission to discharge.

Prior to, during, and post-hospitalisation, topicals were the most frequently used, followed by systemic therapy, phototherapy, and biologics. (Tables 1-2)

The mean (range) LOS was 17.0 (2-71) days. For patients achieving PASI75, the mean LOS was 18.1 vs. 13.1 days for those not achieving PASI75. Multivariate regression analyses suggest that (coefficient estimate, p-value) higher PASI at admission $(0.27,<0.01)$, arterial hypertension $(7.64,0.03)$, and time (months) since diagnosis $(0.02,0.05)$ contributed to longer LOS.

In recent years, there has been a decline in the number of overnight hospital stays for psoriasis. ${ }^{7}$ Treatments received are consistent with NICE and European clinical guidelines recommending phototherapy or systemic therapy in combination with topicals for moderate-tosevere psoriasis. ${ }^{5,8,9}$ Nevertheless, the proportion of patients prescribed biologics at discharge was lower than expected, since $68 \%$ reported a mean PASI or DLQI score $>10$ at discharge after receiving intensive treatment in the inpatient setting. Our inpatient sample may reflect a more

This article is protected by copyright. All rights reserved. 
complex population with difficult-to-control psoriasis, who may be unresponsive or contraindicated to biologics.

Study limitations include the modest sample size, which based on enrolment logs, represented $57 \%$ of patients presenting for hospital stays. Clinical differences between enrolled/non-enrolled patients cannot be determined. Also, given the proportion of missing data in some variables, results should be assessed prudently.

This study demonstrated that disease burden and LOS are substantial among hospitalised psoriasis patients. Whilst on average, patients' psoriasis improved in hospital, they still had suboptimal clinician- and patient-reported outcomes at discharge. Further investigation is required to elucidate parallels between the study group and all psoriasis patients and the low use of biologics in our study. Whilst inpatient psoriasis hospitalisations have declined, this study suggests that it remains an important service for a subgroup of patients. Future research may help to alleviate inpatient disease burden, reduce LOS and avoid future hospital admissions through outpatient treatment options.

\section{REFERENCES}

1. National Institute for Health and Clinical Excellence. Etanercept and Efalizumab for the Treatment of Adults with Psoriasis. NICE Technology Appraisal Guidance 103. 2006; reissued December 2009.

2. National Institute for Health and Care Excellence. Psoriasis: assessment and management. Available at: https://www.nice.org.uk/guidance/cg153/chapter/1-guidance (last accessed 4 December 2015).

3. Gillard SE, Finlay AY. Current management of psoriasis in the United Kingdom: patterns of prescribing and resource use in primary care. Int J Clin Pract 2005; 59: 1260-67.

4. Conway C., Currie C. Descriptive epidemiology of hospitalisation for psoriasis. Curr Med Res Opin 2008; 24: 3487-91.

This article is protected by copyright. All rights reserved. 
5. Woods AL, Rutter KJ, Gardner LS, et al. Inpatient management of psoriasis: a multicentre service review to establish national admission standards. Br J Dermatol 2008; 158: $266-72$.

6. SAS Institute Inc. SAS ${ }^{2}$ 9.3. Cary, NC: SAS Institute Inc.

7. NHS Hospital episode statistics. HES online 2003, 2011, 2012. Available at: www.hesonline.nhs.uk (last accessed 23 January 2013).

8. Pathirana D, Ormerod AD, Saiag P, et al. European S3-guidelines on the systemic treatment of psoriasis vulgaris. J Euro Acad Dermatol Venereol 2009; 23 Suppl 2:1-70.

9. Nast A, Boehncke WH, Mrowietz U, et al. Guidelines on the treatment of psoriasis vulgaris (English version). Update. JDDG: J Dtsch Dermatol Ges 2012; 10: S1-S95.

10. LE Kazis, JJ Anderson, RF Meenan. Effect sizes for interpreting changes in health status. Med Care 1989; 27:S178-S189.

This article is protected by copyright. All rights reserved. 


\section{TABLES}

Table 1: Demographic and clinical characteristics of the patient population at admission.

\begin{tabular}{|c|c|}
\hline Characteristics & Full Analysis Set $(\mathrm{N}=61)^{*}$ \\
\hline Age, mean $(\mathrm{SD})$ years & $45.5(17.1)$ \\
\hline $\begin{array}{l}\text { Gender, n (\%) } \\
\quad \text { Male }\end{array}$ & $31(50.8)$ \\
\hline $\begin{array}{l}\text { Ethnic Group, n (\%) } \\
\text { White } \\
\text { Black } \\
\text { Asian } \\
\text { Other }\end{array}$ & $\begin{aligned} 47 & (95.9) \\
0 & (0.0) \\
1 & (2.0) \\
1 & (2.0)\end{aligned}$ \\
\hline Body Mass Index, mean (SD) kg/m² & $32.1(9.7)$ \\
\hline $\begin{array}{l}\text { Employment Status at Admission, } \mathrm{n}(\%) \\
\text { Unemployed } \\
\text { Change in employment due to psoriasis }\end{array}$ & $\begin{array}{l}37(64.9) \\
22(44.9)\end{array}$ \\
\hline Time Since Psoriasis Diagnosis, mean (SD) years & $20.0(14.2)$ \\
\hline $\begin{array}{l}\text { Previous Hospitalisations for Psoriasis, n (\%) } \\
1 \text { or more previous hospitalisations } \\
2 \text { or more previous hospitalisations } \\
11 \text { or more previous hospitalisations }\end{array}$ & $\begin{array}{l}37(78.7) \\
28(59.6) \\
9(19.1)\end{array}$ \\
\hline Number of Clinician-Diagnosed Comorbid Conditions, mean (SD) & $2.5(1.9)$ \\
\hline $\begin{array}{l}\text { Commonly Reported Comorbid Conditions, n (\%) } \\
\text { Psoriatic arthritis } \\
\text { Depression } \\
\text { Arterial hypertension } \\
\text { High cholesterol } \\
\text { Diabetes } \\
\text { Cardiac disease } \\
\text { Chronic obstructive pulmonary disease } \\
\text { Crohn's or ulcerative colitis } \\
\text { Kidney disease } \\
\end{array}$ & $\begin{array}{c}21(34.4) \\
15(24.6) \\
13(21.3) \\
10(16.4) \\
9(14.8) \\
8(13.1) \\
5(8.2) \\
2(3.3) \\
1(1.6) \\
\end{array}$ \\
\hline $\begin{array}{l}\text { Patient-reported Outcomes, mean (SD) score } \\
\text { SF-12v2 Physical Component Summary } \\
\text { SF-12v2 Mental Component Summary } \\
\text { HADS Anxiety } \\
\text { HADS Depression } \\
\text { WPAI Overall Work Impairment } \\
\text { WPAI Activity Impairment } \\
\end{array}$ & $\begin{array}{c}35.4(10.3) \\
32.1(11.1) \\
9.7(3.0) \\
9.6(2.3) \\
79.2(25.8) \\
68.7(28.4)\end{array}$ \\
\hline
\end{tabular}

This article is protected by copyright. All rights reserved. 


\begin{tabular}{|l|c|}
\hline Characteristics & Full Analysis Set $(\mathbf{N = 6 1})^{*}$ \\
\hline Treatment Types Prescribed 6 Months Prior to Admission, n (\%) & $6(9.8)$ \\
Biologicals & $20(32.8)$ \\
Phototherapy & $26(42.6)$ \\
Systemic Therapy & $56(91.8)$ \\
Topicals & Abbreviations: GCSE/O: general certificate of secondary education/ordinary level; GED: general education diploma; SD: standard deviation \\
\hline
\end{tabular}

*61 patients were enrolled at admission, ethnicity, BMI, employment status, change in employment due to psoriasis, time since psoriasis diagnosis, previous hospitalisations for psoriasis, number of clinician-diagnosed comorbid conditions, SF-12v2, HADS, WPAI Overall Work Impairment and WPAI Activity Impairment, results are available for 49, 59, 57, 49, 60, 47, 50, 45, 58, 18 and 55 patients, respectively.

Table 2: Outcomes and treatment at admission and discharge.

\begin{tabular}{|c|c|c|c|c|c|}
\hline Item & Admission & Discharge & $\begin{array}{l}\text { Change from } \\
\text { Admission to } \\
\text { Discharge* }\end{array}$ & $\begin{array}{c}\text { Standardized } \\
\text { Response } \\
\text { Mean** }\end{array}$ & $\begin{array}{c}\text { p- } \\
\text { value* }\end{array}$ \\
\hline BSA score & & & & -0.95 & $<0.01$ \\
\hline $\mathrm{n}$ & 46 & 34 & 34 & & \\
\hline Mean (SD) & $56.0(27.8)$ & $33.2(26.7)$ & $-24.9(26.3)$ & & \\
\hline 95\% Confidence Interval & $(47.8,64.3)$ & $(23.9,42.5)$ & $(-34.1,-15.8)$ & & \\
\hline Total PASI & & & & -1.01 & $<0.01$ \\
\hline $\mathrm{n}$ & 57 & 35 & 35 & & \\
\hline Mean (SD) & $25.2(15.7)$ & $12.1(9.8)$ & $-14.6(14.5)$ & & \\
\hline 95\% Confidence Interval & $(21.0,29.3)$ & $(8.7,15.5)$ & $(-19.5,-9.6)$ & & \\
\hline Total Average PGA Score & & & & -1.34 & $<0.01$ \\
\hline $\mathrm{n}$ & 45 & 35 & 35 & & \\
\hline Mean (SD) & $3.0(0.7)$ & $1.8(0.9)$ & $-1.1(0.9)$ & & \\
\hline 95\% Confidence Interval & $(2.8,3.2)$ & $(1.5,2.1)$ & $(-1.4,-0.8)$ & & \\
\hline $\begin{array}{l}\text { Number of body part(s) } \\
\text { affected by psoriasis }\end{array}$ & & & & -0.85 & $<0.01$ \\
\hline $\mathrm{n}$ & 58 & 55 & 55 & & \\
\hline Mean (SD) & $9.7(1.7)$ & $6.3(4.1)$ & $-3.4(4.0)$ & & \\
\hline 95\% Confidence Interval & $(9.3,10.1)$ & $(5.2,7.4)$ & $(-4.5,-2.3)$ & & \\
\hline $\begin{array}{l}\text { EQ-5D index (Health State } \\
\text { Utility) }\end{array}$ & & & & 0.55 & $<0.01$ \\
\hline $\mathrm{n}$ & 55 & 47 & 45 & & \\
\hline Mean (SD) & $0.34(0.35)$ & $0.60(0.35)$ & $0.22(0.40)$ & & \\
\hline 95\% Confidence Interval & $(0.24,0.43)$ & $(0.49,0.70)$ & $(0.10,0.34)$ & & \\
\hline DLQI Total Score & & & & -1.03 & $<0.01$ \\
\hline $\mathrm{n}$ & 58 & 47 & 47 & & \\
\hline Mean (SD) & $20.1(7.6)$ & $12.0(8.2)$ & $-8.3(8.0)$ & & \\
\hline $95 \%$ Confidence Interval & $(18.1,22.1)$ & $(9.6,14.4)$ & $(-10.6,-5.9)$ & & \\
\hline \multicolumn{6}{|l|}{$\begin{array}{l}\text { Psoriasis-related Symptom } \\
\text { Scores Due to Psoriasis Over } \\
\text { the Past } 24 \text { Hours }\end{array}$} \\
\hline Worst itching & & & & -1.03 & $<0.01$ \\
\hline$n$ & 58 & 49 & 49 & & \\
\hline Mean (SD) & $7.6(2.5)$ & $4.1(2.9)$ & $-3.3(3.2)$ & & \\
\hline
\end{tabular}

This article is protected by copyright. All rights reserved. 


\begin{tabular}{|c|c|c|c|c|c|}
\hline Item & Admission & Discharge & $\begin{array}{c}\text { Change from } \\
\text { Admission to } \\
\text { Discharge* }\end{array}$ & $\begin{array}{c}\text { Standardized } \\
\text { Response } \\
\text { Mean }\end{array}$ & $\begin{array}{c}\text { p- } \\
\text { value* }\end{array}$ \\
\hline 95\% Confidence Interval & $(6.9,8.2)$ & $(3.3,5.0)$ & $(-4.3,-2.4)$ & & \\
\hline Worst joint pain & & & & -0.40 & 0.01 \\
\hline $\mathrm{n}$ & 58 & 48 & 48 & & \\
\hline Mean (SD) & $5.2(3.3)$ & $3.9(3.5)$ & $-1.1(2.7)$ & & \\
\hline 95\% Confidence Interval & $(4.3,6.1)$ & $(2.9,4.9)$ & $(-1.9,-0.3)$ & & \\
\hline Worst flaking skin & & & & -1.39 & $<0.01$ \\
\hline $\mathrm{n}$ & 58 & 48 & 48 & & \\
\hline Mean $(\mathrm{SD})$ & $7.8(2.8)$ & $2.5(2.8)$ & $-5.2(3.7)$ & & \\
\hline 95\% Confidence Interval & $(7.1,8.6)$ & $(1.7,3.4)$ & $(-6.3,-4.1)$ & & \\
\hline Worst skin burning & & & & -0.86 & $<0.01$ \\
\hline $\mathrm{n}$ & 58 & 48 & 48 & & \\
\hline Mean (SD) & $5.9(3.21)$ & $3.1(3.18)$ & $-3.2(3.66)$ & & \\
\hline 95\% Confidence Interval & $(5.1,6.8)$ & $(2.2,4.0)$ & $(-4.2,-2.1)$ & & \\
\hline Worst redness of skin & & & & -1.10 & $<0.01$ \\
\hline $\mathrm{n}$ & 58 & 49 & 49 & & \\
\hline Mean (SD) & $7.8(2.3)$ & $4.2(2.6)$ & $-3.5(3.2)$ & & \\
\hline $95 \%$ Confidence Interval & $(7.2,8.4)$ & $(3.5,5.0)$ & $(-4.5,-2.6)$ & & \\
\hline Worst skin tightness & & & & -1.07 & $<0.01$ \\
\hline $\mathrm{n}$ & 58 & 49 & 49 & & \\
\hline Mean (SD) & $7.2(2.7)$ & $3.4(3.0)$ & $-3.7(3.4)$ & & \\
\hline 95\% Confidence Interval & $(6.5,7.9)$ & $(2.6,4.3)$ & $(-4.7,-2.7)$ & & \\
\hline Worst bleeding skin & & & & -0.99 & $<0.01$ \\
\hline $\mathrm{n}$ & 58 & 47 & 47 & & \\
\hline Mean (SD) & $4.1(3.2)$ & $0.7(1.0)$ & $-3.1(3.1)$ & & \\
\hline 95\% Confidence Interval & $(3.3,5.0)$ & $(0.4,1.0)$ & $(-4.0,-2.2)$ & & \\
\hline $\begin{array}{l}\text { Treatment Types Prescribed }{ }^{\dagger}, \mathrm{n} \\
(\%)\end{array}$ & & & & N/A & N/A \\
\hline Biologicals & $4(6.6)$ & $5(8.2)$ & N/A & & \\
\hline Phototherapy & $14(23.0)$ & $16(26.2)$ & N/A & & \\
\hline Systemic therapy & $33(54.1)$ & $25(41.0)$ & N/A & & \\
\hline Topicals & $61(100.0)$ & $54(88.5)$ & N/A & & \\
\hline
\end{tabular}

Abbreviations: PGA: physician global assessment; BSA: body surface area; PASI: Psoriasis Area Severity Index; EQ-5D: EuroQoL 5 dimensions questionnaire; DLQI: Dermatology Life Quality Index; PCS: Physical Component Summary Score; MCS: Mental Component Summary Score; WPAI: Work Productivity and Activity Impairment

*61 patients were enrolled at admission. Results are for those with both admission and discharge results.

**Standardised response means were calculated as the mean change from admission to discharge divided by the standard deviation of this change.The following cut-off points may be used loosely to assist with interpretation of effect sizes: 'trivial' $(<0.20)$, 'small' $(\geq 0.20-<0.50)$, 'moderate' $(\geq 0.50-<0.80)$, or 'large' $(\geq 0.80)^{10}$

Biologicals includes: Adalimumab, Etanercept, Infliximab, Ustekinumab, Other biologic; Phototherapy includes: PUVA, Broadband UVB, Narrowband UVB and other phototherapy; Systemic therapy includes: Acitetrin, Ciclosporin, Corticosteroids, Fumeric acid, Methotrexate, Other systemic therapy; Topicals includes: Corticosteroids, Vitamin D, Coal tar, Crude coal tar, High dose calcipotriol, Dithranol and Other topical

This article is protected by copyright. All rights reserved. 